\title{
EFECTO DE DOS INTENSIDADES DE PASTOREO SOBRE LAS PROPIEDADES HIDRÁULICAS DE UN ANDISOL (DURIC HAPLUDAND)
}

\section{EFFECT OF TWO GRAZING INTENSITIES ON THE HYDRAULIC PROPERTIES OF AN ANDISOL (DURIC HAPLUDAND)}

\author{
Carlos Alarcón V. ${ }^{1}$, José Dörner F. ${ }^{1}$, Dorota Dec B. ${ }^{1}$, Oscar Balocchi L. ${ }^{2}$, Ignacio López C. ${ }^{2}$ \\ ${ }^{1}$ Instituto de Ingeniería Agraria y Suelos. Facultad de de Ciencias Agrarias, Universidad Austral de \\ Chile.josedorner@uach.cl \\ ${ }^{2}$ Instituto de Producción Animal. Facultad de de Ciencias Agrarias, Universidad Austral de Chile.
}

\section{ABSTRACT \\ Key words: Grazing intensities, hydraulic properties, Andisol.}

To evaluate the grazing effect on soil hydraulic properties, structural properties of an Andisol under pasture with two grazing intensities were compared. The plots were sown in March 2006 with Lolium perenne cv. Arrow mixed with Trifolium repens cvs. Will and Huia. Before the first grazing, carried out in November 2006, the height of the pasture was homogenised by cutting once. The grazing frequency depended on the numbers of leaves of Lolium perenne cv. Arrow. The cows were removed from the plots when the height of the residue reached 5 or 8 $\mathrm{cm}$ (treatments). In March 2007 undisturbed soil samples were collected. The water retention curve, air permeability and saturated hydraulic conductivity were measured and the pore-size distribution, air-filled porosity, pore-continuity indexes and blocked porosities were calculated.

The results did not show significant differences for any of the soil properties, mainly due to the excellent physical qualities of the Andisol and the season when the samples were collected (lower rainfall and water content in the soil, therefore, greater bearing capacity). Consequently, the Andisol, Serie Valdivia is able to sustain a high grazing intensity during the spring-summer season, without a deterioration of its structural properties and pore functions.

\section{RESUMEN}

Palabras clave: Intensidad de pastoreo, propiedades hidráulicas, Andisol.

Para evaluar el efecto del pastoreo directo sobre las propiedades hidráulicas del suelo, se compararon propiedades estructurales de un suelo bajo pradera con dos intensidades de pastoreo. Las parcelas fueron sembradas en Marzo del 2006 con Lolium perenne cv. Arrow en mezcla con Trifolium repens cvs. Will y Huia. Antes del primer pastoreo, realizado en noviembre de 2006, se realizó un corte de homogenización. La frecuencia de pastoreo se basó en el número de hojas de Lolium perenne cv. Arrow. Las vacas fueron retiradas del sector cuando la pradera alcanzó una altura de residuo de 5 y $8 \mathrm{~cm}$ (tratamientos). En marzo de $2007 \mathrm{se}$ recolectaron muestras no disturbadas de suelo. Se determinó la curva de retención de agua, la permeabilidad de aire y la conductividad hidráulica en fase saturada y se calculó la distribución de poros por tamaño, la porosidad de aire, índice de continuidad de poros y el volumen de poros bloqueados.

Los resultados no evidenciaron diferencias estadísticas significativas para ninguna de las propiedades del suelo analizadas, esto debido principalmente a las excelentes cualidades físicas que presenta el Andisol estudiado y a la temporada del muestreo (menores precipitaciones y contenido de agua en el suelo,

Recepción de originales: 20 de enero 2010. 
por lo tanto, mayor capacidad de soporte). Consecuentemente, el Andisol, Serie Valdivia puede sustentar una alta intensidad de pastoreo en la temporada primavera-verano, sin que esto signifique un deterioro significativo de sus propiedades estructurales ni la función de sus poros.

\section{INTRODUCCIÓN}

En el sur de Chile los sistemas de producción lechera basan principalmente su alimentación en el pastoreo de praderas permanentes, donde las condiciones climáticas durante la primavera y el verano permiten acumular una gran cantidad de forraje (Goic y Matzner, 1977, Balocchi et al., 2002;). Sin embargo, el manejo del pastoreo no siempre se realiza en condiciones que favorezcan un alto rendimiento, persistencia y calidad nutritiva. La baja eficiencia en el uso de las praderas, junto al inadecuado nivel de fertilidad del suelo, son factores importantes que limitan su productividad (Teuber et al., 2008).

El manejo del pastoreo corresponde a un sistema complejo donde interactúan íntimamente los animales, la pradera y el suelo, siendo además afectados por las condiciones climáticas. De esta forma el hombre debe manejar una cantidad de factores para que este sistema sea productivo, eficiente y sobre todo sostenible en el tiempo (Pizzio y Pallares, 2002). Una variable utilizada en el manejo del pastoreo, para controlar la eficiencia de utilización de la pradera corresponde a la intensidad de pastoreo. Esta se relaciona con la cantidad de pradera residual que dejan los animales una vez que esta ha sido pastoreada, por lo que es un índice de medición de la eficiencia de utilización de la pradera que, además, genera una regulación del consumo que realizan los animales (Demanet et $a l .$, 2007). A medida que aumenta la altura de residuo (baja intensidad de pastoreo), aumenta el consumo de forraje por animal, producto de la menor presión de pastoreo. Como consecuencia de ello, sin embargo, disminuye la eficiencia de la utilización de la pradera y la calidad de los rebrotes subsiguientes. Por el contrario, un pastoreo intensivo, además de reducir severamente el consumo y rendimiento animal individual, deja un residuo muy escaso que es insuficiente para sostener un rebrote vigoroso (Parga, 2006).

El efecto del pastoreo intensivo sobre el suelo es mencionado por autores como Blaser (1966), Willatt y Pullar (1983) y Greenwood y Mckenzie (2001). Ellos señalan que el pastoreo intensivo produce un incremento en la densidad del suelo, una disminución en la porosidad y continuidad entre los poros y una reducción de la estabilidad de agregados, provocando, además, la eliminación de las especies pratenses lo que ocasiona un aumento en la susceptibilidad a la erosión. También se debe considerar que un aumento en la intensidad de pastoreo puede disminuir el contenido de agua en el suelo específicamente en el horizonte A. Esto depende principalmente del tipo de suelo, ya que existen situaciones donde una mayor intensidad de pastoreo no provoca modificaciones en las propiedades físicas del suelo (Donkor et al., 2002).

La compactación del suelo ocurre cuando es sobrepasada su capacidad de soporte lo que genera un aumento en la densidad aparente, produciéndose una redistribución del volumen poroso, lo que afecta su funcionalidad expresada a través de la capacidad para conducir agua y aire (Hartge y Horn, 1991, Hillel, 1998, Dörner y Horn, 2006). La resistencia mecánica del suelo varía en función de la tensión con que está retenida el agua en los poros, ya que la capacidad de soporte depende en gran medida de la forma de los meniscos que se forman en torno a los puntos de contacto, en donde predominan las uniones sólido-agua-sólido (Hartge, 2000). Es así como el pastoreo en altas condiciones de humedad puede provocar un deterioro de tipo estructural en el suelo, ya que la presión que generan los animales bovinos (hasta $192 \mathrm{kPa}$, 
según Taboada, 2007), supera la capacidad de soporte del suelo (49 kPa en un Typic Hapludand Serie Pelchuquín según Dörner et al., 2009a). Por otro lado, la disminución en el contenido de agua en el suelo (lo que generalmente ocurre en la época de verano) y el consecuente incremento en la capacidad de soporte ( $>220 \mathrm{kPa}$ según Ellies, 1988) permiten sustentar la presión y así evitar la deformación del suelo.

De acuerdo a lo anterior, el objetivo de este trabajo fue evaluar el efecto de un aumento en la intensidad de pastoreo con vacas lecheras, sobre algunas propiedades físicas de un Andisol (Duric Hapludand) de la Región de los Ríos en la temporada de primavera - verano.

\section{MATERIAL Y MÉTODO}

\section{Suelo, precipitaciones y manejo de pastoreo}

El suelo donde se establecieron las praderas correspondió a un Andisol serie Valdivia (Duric Hapludand, CIREN, 2003). Estos suelos son denominados Trumaos en el sur de Chile.

El estudio se realizó en la Estación Experimental Santa Rosa de la Universidad Austral de Chile, coordenadas UTM* ${ }^{*}, 5.594 .808$ m Norte y 651.625 m Este, a una altitud 12 m.s.n.m. Las parcelas fueron sembradas el 17 de Marzo de 2006 con ballica inglesa (Lolium perenne $\mathrm{cv}$. Arrow $25 \mathrm{~kg} \mathrm{ha}^{-1}$ ) en mezcla con dos variedades de trébol blanco (Trifolium repens cv. Will, $2 \mathrm{~kg} \mathrm{ha}^{-1}$ y Trifolium repens cv. Huia, $\left.2 \mathrm{~kg} \mathrm{ha}^{-1}\right)$. Antes de realizar el primer pastoreo, el día 27 de Octubre de 2006, se realizó un corte de homogenización a la pradera con el objetivo de que las especies pratenses tengan una altura similar al momento de comenzar a pastorear. El primer pastoreo con animales se realizó el día 13 de Noviembre de 2006.

El manejo del pastoreo para el experimento consistió en seleccionar al azar un número de 7 vacas de la lechería del predio (Frisón negro, con un peso entre 500 a $600 \mathrm{~kg}$ ). Las vacas provenían de la primera ordeña del día (en la mañana), las que eran llevadas a pastorear al predio experimental en donde entraban a una parcela de $200 \mathrm{~m}^{2}$. El criterio de entrada para que los animales pastoreen en las parcelas, se determinó mediante la frecuencia de pastoreo. Esta se encuentra determinada por el número de hojas de la ballica inglesa (Lolium perenne cv. Arrow), que para éste caso fue de 2,5 hojas por macollo. Posteriormente, los animales fueron retirados del sector cuando la pradera logró alcanzar una altura de residuo provocada por la defoliación de los animales de 5 y $8 \mathrm{~cm}$. La carga animal instantánea utilizada en el pastoreo fue equivalente a 350 vacas por hectárea. El tiempo necesario para alcanzar la altura de residuo de $5 \mathrm{~cm}$ fue de aproximadamente 8 horas, y para la altura de residuo de $8 \mathrm{~cm}$ se necesitaron de 5 horas aproximadamente.

Finalmente, con el fin de caracterizar la distribución de precipitaciones de un año normal en la zona en estudio y en especial durante la estación primavera-verano, se analizaron datos diarios de precipitaciones de 20 años de estudios, proporcionado por la Estación Meteorológica de la Universidad Austral de Chile. Mayores detalles respecto a los cálculos realizados se encuentran en Alarcón (2008).

\section{Recolección de muestras de suelo.}

El muestreo de suelo se realizó el 15 de marzo de 2007. Los sectores donde se procedió a tomar las muestras para el análisis físico correspondieron a parcelas con frecuencias de pastoreo de 2,5 hojas por macollo. Por lo tanto, el experimento consideró dos tratamientos (5 y $8 \mathrm{~cm}$ de altura de residuo) con tres repeticiones. Se recolectaron un total de 96 muestras no disturbadas (cilindros de $250 \mathrm{~cm}^{3}$ ) del horizonte superficial del suelo $(0-10 \mathrm{~cm}$ de profundidad). Del total de cilindros, 36 se utilizaron para elaborar la curva de retención de agua, específicamente 6 cilindros por parcela. Los otros 60 cilindros restantes se usaron para determinar la conductividad hidráulica en fase saturada (Ks), específicamente 10 cilindros por parcela.

\section{Análisis de laboratorio \\ Caracterización general del suelo.}

El análisis textural se realizó utilizando

* coordenadas referidas al elipsoide internacional, datum sudamericano 1956. 
el método de Day (Forsythe, 1974). Para la determinación del contenido de materia orgánica se utilizó el método de digestión húmeda, también se determinó el $\mathrm{pH}$ al agua y $\mathrm{pH}$ al cloruro de calcio, todos basados en la metodología descrita por Sadzawka (2006).

\section{Determinación de la curva de retención de agua.}

Las muestras no disturbadas de suelo, fueron saturadas por medio de ascenso capilar de agua con el objetivo de evitar inclusiones de aire en los poros del suelo. La saturación tomó aproximadamente cuatro días. Posteriormente, las muestras fueron equilibradas a los potenciales mátricos de: $-10,-20$ y $-30 \mathrm{hPa}$ en bandejas de arena, $-60,-150,-330,-500$ y 15430 hPa por medio de platos cerámicos contenidos en una olla de presión. Una vez que las muestras alcanzaron un equilibrio con el potencial mátrico aplicado, se determinó el contenido gravimétrico de agua por medio de pesaje (Hartge y Horn, 1991). Para determinar la densidad aparente, las muestras se secaron en una estufa a $105^{\circ} \mathrm{C}$ por 24 horas (Forsythe, 1974). La densidad real se midió de acuerdo al método del pesaje sumergido (Forsythe, 1974). A partir de la curva $\mathrm{pF}$ se determinó la distribución de poros del suelo por medio de la diferencias en el contenido volumétrico de agua entre $0-60 \mathrm{hPa}$ ( $\phi$ poros $>50 \mu \mathrm{m}), 60-330 \mathrm{hPa}$ $(\phi$ poros $50-10 \mu \mathrm{m}), 330-15430 \mathrm{hPa}(\phi$ poros $10-2 \mu \mathrm{m})$ y el contenido volumétrico de agua a $15430 \mathrm{hPa}(\phi$ poros $<2 \mu \mathrm{m})$.

\section{Determinación de la permeabilidad de aire.}

La permeabilidad de aire $(\mathrm{Ka})$ se midió durante la determinación de la curva $\mathrm{pF}$, específicamente a potenciales mátricos de $-60,-150,-330,-500$ $\mathrm{hPa}$ utilizando un permeámetro de aire. Esta medición consistió en hacer circular aire con una presión menor a $1 \mathrm{hPa}$, en forma ascendente a través de la muestra de suelo, con el objetivo de permitir y conservar un flujo laminar. La determinación de la permeabilidad de aire $\mathrm{Ka}$ $\left[\mu \mathrm{m}^{2}\right]$ se calculó a partir de la conductividad de aire $\mathrm{Kl}\left[\mathrm{cm} \mathrm{s}^{-1}\right]$ mediante la siguiente ecuación.

$$
K_{a}=K_{l} \cdot \frac{\eta}{\rho_{l} \cdot g}
$$

Donde $\eta$ es la viscosidad del aire $\left[\mathrm{g} \mathrm{s}^{-1} \mathrm{~cm}^{-1}\right]$, $\mathrm{P}_{1}$ es la densidad del aire durante la medición $\left[\mathrm{kg} \mathrm{m}^{-3}\right]$ y g la aceleración de gravedad 9,81 [m $\mathrm{s}^{-2}$. Además, se determinó la fracción de poros llenos con aire $\varepsilon_{\mathrm{a}}\left[\mathrm{cm}^{3} \mathrm{~cm}^{-3}\right]$ a través de la curva de retención de agua, como la diferencia entre la porosidad total y el con tenido volumétrico a un determinado potencial mátrico (Dörner y Horn, 2006).

A partir de $\mathrm{K}_{\mathrm{a}}$ y $\varepsilon_{\mathrm{a}}$ se calcularon índices de continuidad de poros con el objetivo de avaluar aspectos funcionales del medio poroso. Uno de ellos, fue el propuesto por Groenevelt et al. (1984), que se calcula a partir de la siguiente ecuación:

$K_{1}=\frac{K_{a}}{\varepsilon_{a}}(2)$

Otro índice que permite caracterizar la geometría del medio poroso, se puede derivar de la relación entre $\mathrm{K}_{\mathrm{a}}$ y $\varepsilon_{\mathrm{a}}$ tal como lo proponen Ball et al. (1988).

$\log \left(\mathrm{K}_{\mathrm{a}}\right)=\log (\mathrm{M})+\mathrm{N} \log \left(\varepsilon_{\mathrm{a}}\right)$

Donde $\mathrm{M}$ y $\mathrm{N}$ son parámetros empíricos. $\mathrm{N}$ es un índice de continuidad de poros que refleja el incremento de la permeabilidad del aire en función del aumento de los poros con aire (Ahuja et al., 1984, Ball et al., 1988). El mismo parámetro representa la disminución de la tortuosidad de los poros y del área superficial con el incremento de la fracción de poros disponibles para el flujo.

Un índice que permite estimar el volumen de poros bloqueados $\left(\varepsilon_{\mathrm{b}}\right)$ que no participan del flujo de aire por convección fue propuesto por Ball et al. (1988). Ellos indican que suelos que presentan una permeabilidad de aire igual o menor a $1 \mu \mathrm{m}^{2}$ pueden ser considerados como impermeables; por lo tanto, el intercepto en la abscisa corresponde a un estimador del volumen de poros bloqueados.

$$
\varepsilon_{b}=10^{(-\log M) / N}\left[\mathrm{~cm}^{3} \mathrm{~cm}^{-3}\right]
$$


Cuadro 1: Distribución quincenal de las precipitaciones.

Table 1: Fortnightly rainfall distribution

\begin{tabular}{|c|c|c|c|}
\hline Fecha & Precipitación [mm] & Fecha & Precipitación [mm] \\
\hline 1 - 14 Enero & 0,1 & $2-15$ Julio & 64,6 \\
\hline 15 - 28 Enero & 0 & 16 - 29Julio & 79,2 \\
\hline 29 Enero - 11Febrero & 0 & 30 Julio -12 Agosto & 42,5 \\
\hline 12 - 25 Febrero & 0 & 13 - 26 Agosto & 45,8 \\
\hline 26 Febrero -11 Marzo & 0,4 & 27 Agosto -9 Septiembre & 48,2 \\
\hline 12 - 25 Marzo & 0,6 & $10-23$ Septiembre & 23,3 \\
\hline 26 Marzo - 8 Abril & 2,9 & 24 Septiembre -7Octubre & 3,2 \\
\hline 9 - 22 Abril & 15 & 8-21 Octubre & 13,3 \\
\hline 23 Abril - 6Mayo & 11 & 22 Octubre - 4 Noviembre & 8,7 \\
\hline 7 - 20 Mayo & 24 & 5-18 Noviembre & 3,2 \\
\hline 21Mayo - 3 Junio & 58,1 & 19 Noviembre -2 Diciembre & 2,7 \\
\hline 4 -17Junio & 90,5 & 3 Diciembre -16 Diciembre & 2 \\
\hline 18 Junio - 1 Julio & 118,5 & 17 - 31 Diciembre & 0 \\
\hline
\end{tabular}

Para el cálculo de la distribución de las precipitaciones se usaron datos diarios aportados por la estación meteorológica de la Universidad Austral de Chile. Otoño: 21 marzo - 21 junio; Invierno: 21 junio - 21 septiembre; Primavera: 21 septiembre - 21 diciembre; Verano: 21 diciembre - 21 marzo.

\section{Determinación de la conductividad hidráulica saturada}

Para la medición de la conductividad hidráulica en fase saturada (Ks) se utilizó un permeámetro de carga constante. Los cilindros con las muestras no disturbadas de suelo fueron saturados por ascenso capilar de agua por 48 horas. Posteriormente, se hizo circular agua a través de las muestras durante 1 hora, al cabo del cual se determinó el volumen de agua por unidad de tiempo lo que permite obtener valores absolutos de conductividad hidráulica. Manteniendo una circulación de agua constante por las muestras, se realizó una segunda medición a las 6 horas, una tercera medición a las 12 horas y, finalmente, una última medición a las 24 horas de iniciada esta evaluación. Para todas las mediciones se realizaron tres repeticiones por muestra. Posteriormente, se determinó $\mathrm{Ks}\left[\mathrm{cm} \mathrm{d}^{-1}\right]$ de acuerdo a la ley de Darcy, mediante la siguiente ecuación (Eijkelkamp Agrisearch Equipment, 2003).

$\mathrm{Ks}=\frac{V}{A^{*} t} * \frac{L}{h}=\frac{Q^{*} L}{h^{*} A}$

En donde V correspondió al volumen de agua percolada $\left[\mathrm{cm}^{3}\right]$, t el tiempo para alcanzar ese volumen [s], Q es el caudal de agua percolada $\left[\mathrm{cm} \mathrm{s}^{-1}\right]$, L es el largo del cilindro [cm], h es la carga hidráulica $[\mathrm{cm}]$ y A el área del cilindro $\left[\mathrm{cm}^{2}\right]$.

\section{Análisis estadístico.}

El diseño del experimento correspondió al de bloques completos al azar. Los tratamientos fueron las alturas de residuo. Los resultados se analizaron estadísticamente mediante un análisis de varianza (p 0,05), utilizando el programa Statgraphics Plus 5.1.

Los parámetros de la ecuación (3) fueron comparados entre tratamientos utilizando el programa Statgraphics Plus 5.1 (p 0,05). Además, con el mismo paquete estadístico, se realizó la comparación de supuestos que incluyó el test de normalidad de Chi-cuadrado y el test para la homogeneidad de varianza Contraste de Bartlett.

\section{RESULTADOS}

\section{Precipitaciones y características generales del suelo}

El Cuadro 1 presenta la distribución de precipitaciones de 20 años para la ciudad de Valdivia. Las lluvias en la temporada de primavera van disminuyendo considerablemente hasta llegar a la época de verano donde las precipitaciones son casi imperceptibles, y por el contrario en la temporada otoño-invierno 
Cuadro 2: Propiedades generales del suelo.

Table 2: General properties of the soil.

\begin{tabular}{|c|c|c|c|c|c|c|}
\hline $\begin{array}{l}\text { Intensidad } \\
\text { de pastoreo } \\
{[\mathrm{cm}]}\end{array}$ & $\begin{array}{l}\text { Arena } \\
2000-63\end{array}$ & $\begin{array}{c}\operatorname{Limo} \\
63-2 \\
{[\mu \mathrm{m}]}\end{array}$ & $\underset{<2}{\text { Arcilla }}$ & $\begin{array}{c}\text { MOS } \\
{[\%]}\end{array}$ & $\begin{array}{c}\text { pH } \mathbf{H}_{2} \mathbf{0} \\
{[-]}\end{array}$ & $\begin{array}{c}\text { pH } \mathrm{CaCl}_{2} \\
{[-]}\end{array}$ \\
\hline 5 & 16 & 63 & 21 & $17,2 \pm 0,4$ & 5,7 & 5,3 \\
\hline 8 & 16 & 63 & 21 & $15,8 \pm 0,6$ & 5,7 & 5,3 \\
\hline
\end{tabular}

es donde se concentran los mayores eventos. Lo anterior incide en el contenido de agua del suelo.

Algunas características del suelo se presentan en el Cuadro 2. El suelo presenta una clase textural franco-limosa lo que indica una distribución similar entre arena y arcilla pero destacando principalmente el contenido de limo. Los valores promedio de $\mathrm{pH}$ del suelo no presentaron diferencias entre los dos tratamientos. Para el caso del contenido promedio de materia orgánica del suelo (MOS), el tratamiento de mayor intensidad de pastoreo ( $5 \mathrm{~cm}$ de altura de residuo) presentó un contenido MOS ligeramente superior al tratamiento de 8 $\mathrm{cm}$.

Efecto de la intensidad de pastoreo sobre la densidad aparente, espacio poroso y distribución de la porosidad.

Tanto la densidad aparente (Da) como la porosidad total del suelo (o espacio poroso, PT) no se vieron afectados significativamente por el incremento en la intensidad de pastoreo (Cuadro 2). La distribución de los poros (obtenida a partir de la curva $\mathrm{pF}$ o de retención de agua), no presentó diferencias estadísticamente significativas entre los tratamientos, existiendo sólo leves diferencias numéricas entre los distintos tipos de poros.

Efecto de la intensidad de pastoreo sobre

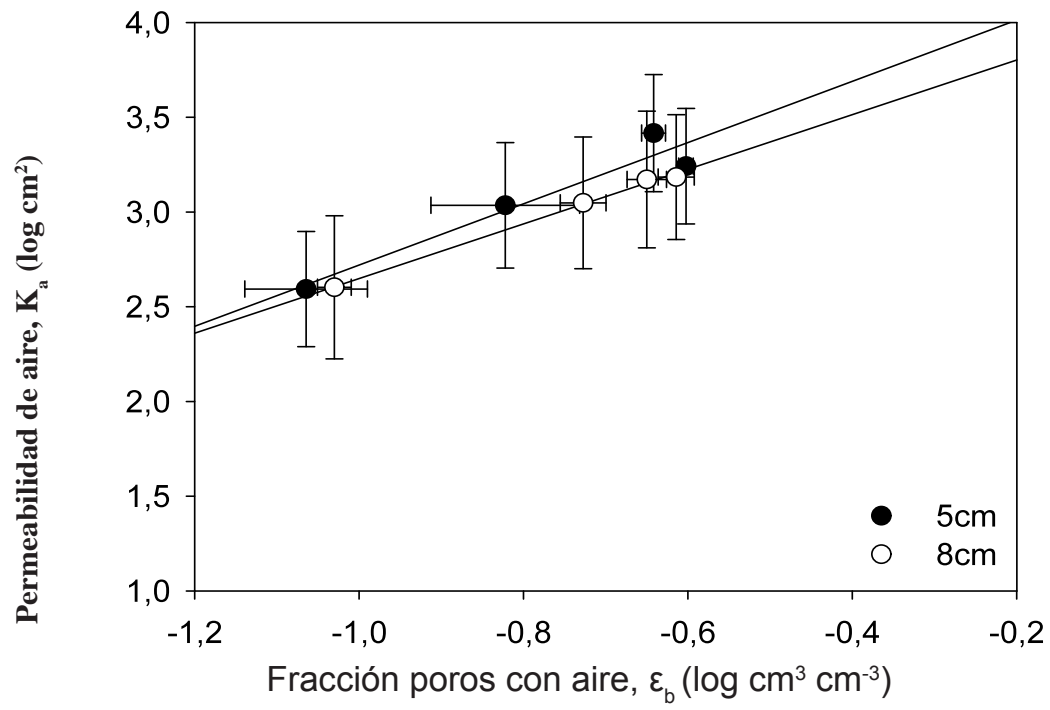

Figura 1: Permeabilidad de aire en función del volumen de poros llenos con aire para las dos intensidades de pastoreo. Las barras de error indican \pm 1 error estándar $(n=3)$.

Figure 1: Air permeability as function of air-filled porosity for both grazing intensities. Bars indicate \pm 1 standard error $(n=3)$. 
Cuadro 3: Efecto de la intensidad de pastoreo sobre la densidad aparente (Da), porosidad total (PT) y su distribución.

Table 3: Effect of grazing intensity on bulk density (Da), total porosity (PT) and its distribution.

\begin{tabular}{lcccccc}
\hline $\begin{array}{c}\text { Intensidad de } \\
\text { pastoreo } \\
{[\mathrm{cm}]}\end{array}$ & Da & PT & $\begin{array}{c}\text { PDR } \\
>50 \mu \mathrm{m}\end{array}$ & $\begin{array}{c}\text { PDL } \\
50-10 \mu \mathrm{m}\end{array}$ & $\begin{array}{c}\text { PAU } \\
10-2 \mu \mathrm{m}\end{array}$ & $\begin{array}{c}\text { PAI } \\
{[0,2 \mu \mathrm{m}}\end{array}$ \\
\hline 5 & {$\left[\mathrm{~g} \mathrm{~cm}^{-3}\right]$} & & {$[\%]$} & {$[\%]$} & {$[\%]$} & {$[\%]$} \\
8 & $0,69 \pm 0,01$ & $70,2 \pm 0,6$ & $9,3 \pm 0,4$ & $13,9 \pm 0,7$ & $21,4 \pm 0,7$ & $25,6 \pm 0,3$ \\
& $0,68 \pm 0,01$ & $70,5 \pm 0,4$ & $9,4 \pm 0,4$ & $13,1 \pm 0,6$ & $22,7 \pm 0,5$ & $25,5 \pm 0,2$ \\
\hline
\end{tabular}

PDR: Poros drenaje rápido, PDL: Poros drenaje lento, PAU: Poros de agua útil, PAI:

Poros de agua inútil. Letras distintas indica diferencias significativas entre los

tratamientos $(\mathrm{p}<0,05)$. Valores promedio \pm 1 error estándar.

la permeabilidad de aire del suelo e índices de continuidad

La Figura 1 muestra la permeabilidad de aire $\left(\mathrm{K}_{\mathrm{a}}\right)$ en función de la fracción de poros saturados con aire $\left(\varepsilon_{\mathrm{a}}\right)$ para la intensidad de pastoreo de 5 y $8 \mathrm{~cm}$ de altura de residuo. La relación entre $\mathrm{K}_{\mathrm{a}}$ y $\varepsilon_{\mathrm{a}}$ se ajustó de acuerdo a la ecuación propuesta por Ball et al. (1988) cuyos parámetros se presentan en el Cuadro 4. La permeabilidad aumenta a medida que aumenta el volumen de poros que están ocupados por aire. Al realizar el análisis estadístico de comparación de las rectas se aprecia, que no hubo diferencias estadísticamente significativas tanto entre la pendiente de las dos rectas como en el intercepto ( $p>0,05)$.

En cuanto a los índices de continuidad de poros $\varepsilon_{\mathrm{b}}$ y $\mathrm{K}_{1}$, se observó que para el caso del volumen de poros bloqueados no se presentaron diferencias estadísticamente significativas como

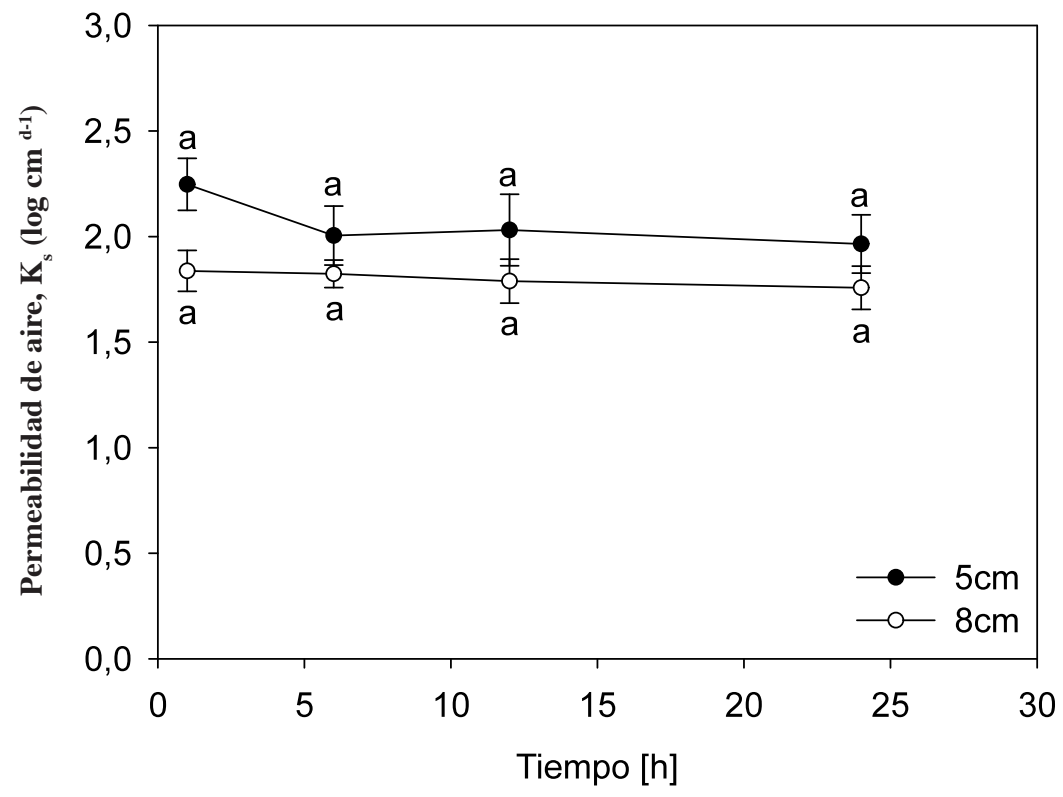

Figura 2: Conductividad hidráulica en función del tiempo para las dos intensidades de pastoreo. Las barras de error indican \pm 1 error estándar $(n=3)$.

Figure 2: Hydraulic conductivity as function of time for both grazing intensities. Bars indicate \pm 1 standard error $(n=3)$. 
consecuencia del incremento en la intensidad de pastoreo. Algo similar ocurrió para el caso de $\mathrm{K}_{1}$.

Efecto de la intensidad de pastoreo sobre la conductividad hidráulica en fase saturada.

En la Figura 2 se presenta el efecto de las dos intensidades de pastoreo sobre la conductividad hidráulica en fase saturada (Ks). Del gráfico se desprende que no existieron diferencias estadísticamente significativas entre las dos intensidades de pastoreo durante todo el ensayo.

\section{DISCUSIÓN}

\section{Características generales del suelo}

Los valores de $\mathrm{pH}$ del suelo al agua (Cuadro 2), son entre 2 y 5 décimas más altos de acuerdo a lo descrito por Rodríguez (1993), quién señala que suelos Trumaos (o Andisoles) de la Región de los Ríos presentan valores promedio de $\mathrm{pH}$ al agua $(1: 2,5)$ de 5,2 a 5,5. Este aumento se debe a la incorporación de cal y tiene por objetivo favorecer un buen desarrollo de las especies pratenses que componen la pradera, las cuales necesitan de un $\mathrm{pH}$ entre 5,4 y 5,8 para las ballicas y para el trébol blanco entre 5,8 y 6,2 (Suárez, 2003).

El contenido de materia orgánica promedio se asemeja a lo descrito por Rodríguez (1993), quién señala que los suelos de la Región de los Ríos presentan contenidos promedio de materia orgánica que fluctúan entre 13,70 y 31,24 (g MOS 100g-1 de suelo), algo característico en los Andisoles. Altos niveles de MOS son asociados con buenas propiedades físicas, debido al aumento de la actividad biológica, la cual se encarga de moldear y agregar las partículas que componen el suelo. Esto es corroborado por Chan y Pratley (1998) y Southorn y Cattle (2004), quienes señalan que suelos con altos contenidos de materia orgánica benefician la agregación y estabilidad del suelo. Algunos autores van más allá, indicando que la MOS, contribuye a una reducción de la densidad aparente, mejora la conductividad hidráulica, la infiltración y retención de agua gracias a la fauna presente en el suelo (Alegre et al., 1996). Por otro lado, la materia orgánica también permite que el suelo mantenga su integridad funcional, ya que actúa como un elemento elástico que permite la recuperación del suelo después de un evento de presión como el pisoteo animal (Dörner et al., 2009b).

\section{Efecto de la intensidad de pastoreo sobre la estructura del suelo.}

$\mathrm{El}$ incremento en la intensidad de pastoreo (de 8 a $5 \mathrm{~cm}$ altura residuo) no significó un aumento en la densidad aparente, demostrando que el suelo fue capaz de soportar el tránsito y pisoteo animal sin producir una deformación significativa del volumen del suelo. Según lo mencionado por Drewry et al.(2000), la densidad aparente puede ser insensible a pequeños cambios en la compactación del suelo, lo que podría expresar el por qué no se han presentado cambios en la densidad aparente, considerando además la etapa inicial de este estudio, en donde los animales pastorearon por un periodo aproximado de cuatro meses. Los valores obtenidos de espacio poroso, que no mostraron diferencias estadísticamente significativas entre los tratamientos, son similares a los descrito por Ellies et al. (2000), quién menciona que los suelos de origen volcánico tienen un espacio poroso promedio de $69 \%$.

Con respecto a la distribución de la porosidad, el volumen de poros de drenaje rápido (PDR) en los tratamientos de 5 y $8 \mathrm{~cm}$ de intensidad de pastoreo (Cuadro 3), no superaron el 10\%, valores que se pueden considerar bajos para el tipo de suelo. Velásquez (1983) determinó en un Andisol serie Valdivia bajo pastoreo valores promedio para PDR de 15\% (0 - 10 $\mathrm{cm}$ de profundidad). Esta diferencia en PDR se puede asociar al manejo y uso constante en el tiempo de éste suelo, el cual durante los últimos 10 años o más, siempre ha sido utilizado para pastoreo directo. Además Nissen et al. (2006) determinaron valores de PDR de 9,3\% en un suelo Trumao (Typic Hapludand) serie Osorno bajo pradera, y de 23,9\% en el mismo suelo bajo bosque. Esto indica, que suelos en condiciones no intervenidas por el hombre, como es el caso del bosque, tienden a presentar una mayor proporción de poros gruesos que suelos utilizados para producción pecuaria y cultivos agrícolas tradicionales. Así la labranza convencional y el manejo intensivo estarían asociados al deterioro de la estructura, al disminuir los poros gruesos 
(macroporos) y aumentar los poros más finos, y consecuentemente, disminuyendo la velocidad de entrada de agua al perfil del suelo, restringiendo de esta manera la aireación (Baver y Gardner, 1973). La disminución de los PDR no deja de ser importante ya que, autores como Grable (1971), Cannell (1977), Carter (1988) y Greenwood y McNamara (1992), mencionan que una macroporosidad menor al $10 \%$ indica limitaciones para las condiciones de salud de las plantas y la aireación del suelo.

Los poros de drenaje lento (PDL) son levemente mayores en comparación a lo determinado por Velásquez (1983), quién para un Andisol de la serie Valdivia sometido a pastoreo, determinó valores de PDL entre un $8 \%$ y $12 \%$. Nissen et al. (2006) determinaron valores de PDL de 10 y $13 \%$ en un Typic Hapludand (serie Osorno) bajo pradera y bosque, respectivamente. Al comparar el valor obtenido en bosque por Nissen et al. (2006) con los resultados determinados en este experimento, se aprecia que son valores bastante cercanos y similares lo que indicaría que su proporción no ha sido tan afectada como el caso de los PDR.

El porcentaje de poros de agua útil (PAU) que el suelo presentó es mayor en comparación a lo mencionado por Vyhmeister (1980), quien determinó para un Andisol de la serie Valdivia un valor de $14 \%$ en una pradera de la misma estación experimental del presente experimento. Además, Nissen $e t$ al. (2006) determinaron para un Andisol bajo bosque y pradera, valores de PAU de 19,5\% y un $14,6 \%$, respectivamente. Dichos valores son menores a los obtenidos en este estudio, lo que fundamenta que un uso agropecuario constante en el tiempo de un mismo sitio específico modificaría la proporción de poros hacía un incremento considerable los PAU. A la misma conclusión llegaron Dörner et al. (2009b) en un Andisol de la serie Pelchuquín pastoreado por 50 años en comparación al mismo suelo pero bajo renoval de bosque nativo.

En cuanto a los poros de agua inútil (PAI), estos son menores a lo descrito por Ellies
(1986) y (1988), donde determinó un 29,2\% y $28,1 \%$ de PAI en un suelo de la serie Valdivia, respectivamente. Esta diferencia se debe al menor contenido de arcilla determinado en este experimento (21\%) en comparación con los resultados obtenidos por Ellies (1986) y (1988), donde el contenido de arcilla era de $34 \%$.

Efecto de la intensidad de pastoreo sobre aspectos funcionales del suelo: permeabilidad de aire y conductividad hidráulica.

Para Bear (1972) la permeabilidad está estrechamente relacionada con factores geométricos del sistema poroso del suelo como la porosidad total, la distribución de los poros por tamaño, la forma del sistema poroso, la continuidad y tortuosidad de los poros. Por lo tanto, la permeabilidad de aire permite caracterizar el sistema poroso funcionalmente (Dörner y Dec, 2007).

No se encontraron mayores diferencias de permeabilidad de aire entre los tratamientos. Sin embargo, es esperable que a futuro la $\mathrm{Ka}$ fuera el primer indicador en expresar diferencias debido a la intensidad de pastoreo. Autores como Greenwood y McKenzie (2001) señalan que la permeabilidad de aire es una medida de continuidad de poros que es más sensible a los cambios estructurales del suelo en comparación a otras propiedades como densidad aparente, resistencia mecánica del suelo, etc.

Los altos valores del índice de continuidad de poros $\mathrm{K}_{1}$ (ecuación 2) observados en ambos tratamientos indican que el medio poroso tiene una alta capacidad para transmitir fluidos debido a una gran continuidad entre los poros (Dörner y Dec, 2007). Esto no deja de ser importante ya que la continuidad y tortuosidad de los poros influyen en el sistema de transporte de agua, solutos y gases en el suelo (Moldrup et al., 2001). Por otro lado, la determinación del volumen de poros bloqueados (Cuadro 4) propuesta por Ball et al. (1988), indican que ambos tratamientos presentan igual volumen de poros dispuestos a contribuir en el flujo de aire en el suelo por convección.

Otro aspecto funcional a considerar es la capacidad que tiene el suelo para conducir agua. Es así que determinaciones de conductividad hidráulica saturada (Ks) han sido utilizadas para caracterizar la estructura de los suelos 
Cuadro 4: Índices de continuidad de poros derivados de la relación entre la permeabilidad de aire $\left(K_{\mathrm{a}}\right)$ y fracción de poros llenos con aire (عa) para las dos intensidades de pastoreo.

Table 4: Pore continuity indexes derived from the relationship between air permeability $\left(K_{a}\right)$ and airfilled porosity (ca) for both grazing intensities.

\begin{tabular}{cccccc}
\hline \multicolumn{7}{c}{$\log \mathrm{K}_{\mathrm{a}}=\log \mathrm{M}+\mathrm{N} \log \left(\varepsilon_{\mathrm{a}}\right)$} & & \\
\hline $\begin{array}{c}\text { Intensidad de } \\
\text { Pastoreo } \\
{[\mathrm{cm}]}\end{array}$ & $\log \mathrm{M}$ & $\mathrm{N}$ & $\mathrm{r}$ & $\varepsilon_{\mathrm{b}}$ & $\mathrm{K}_{1}(60 \mathrm{hPa})$ \\
\hline 5 & {$[-]$} & {$[-]$} & {$[-]$} & {$[\%]$} & {$[-]$} \\
\hline 8 & $4,34 \mathrm{a}$ & $1,63 \mathrm{a}$ & 0,95 & $0,22 \mathrm{a}$ & $30 \mathrm{a}$ \\
\hline
\end{tabular}

Los valores de cada columna con igual letra son estadísticamente similares $(\mathrm{PO}, 05)$

volcánicos, su estabilidad (Ellies et al., 1997) y funcionalidad (Dörner et al., 2009b). Ks depende del monto de poros gruesos y de la forma y continuidad del sistema poroso, que varía fuertemente de un sitio a otro difiriendo además en las distintas orientaciones del suelo ya sea vertical u horizontal (Ellies, 1997; Dörner y Horn, 2006). Ks de la intensidad de 5 $\mathrm{cm}$ es numéricamente mayor a la otra intensidad durante todo el experimento, sin embargo, estas diferencias no son estadísticamente significativas (24 horas; Figura 3). Llama la atención la notable variación de un $11 \%(2,25$ $\log \mathrm{cm} \mathrm{día}{ }^{-1}-2,00 \log \mathrm{cm}$ día-1) que presentó Ks para el tratamiento de mayor intensidad de pastoreo entre la primera y segunda hora de medición. Esto podría explicarse de acuerdo a que inicialmente podrían haber participado bioporos formados por el sistema radical de las especies pratenses y algunas lombrices de tierra. Estos poros tienden a ocluirse por el efecto erosivo del agua que termina cambiando y modificando la estructura, lo que se expresa en una disminución del movimiento de agua en el suelo. Esto es mencionado por Ellies et al. (1997) y Janssen et al. (2004), quienes explican que existe una variación durante el flujo continuo de agua debido oclusiones o aperturas que provocan cambios en la continuidad del sistema poroso. Ellies (2003) determinó valores de Ks en múltiples investigaciones realizadas en suelos bajo distintos usos como por ejemplo: pradera artificial, Ks de $250 \mathrm{~cm} \mathrm{~d}^{-1}$ y pradera degradada, $\mathrm{Ks} 80 \mathrm{~cm} \mathrm{~d}^{-1}$. Los valores determinados en el presente experimento se asemejan a los de una pradera degradada, esto principalmente debido al uso continuo de pradera en el tiempo.

Ante los antecedentes presentados, se desprende que el suelo en la temporada primavera-verano fue capaz de soportar la carga animal, sin que ocurra una alteración estructural significativa con el incremento en la intensidad de pastoreo debido a que la capacidad de soporte aumenta con el menor contenido de humedad del suelo (ver precipitaciones en Cuadro 1) llegando a valores que son superiores (220 kPa, según Ellies, 1988) a la presión (hasta $192 \mathrm{kPa}$ según Taboada, 2007) que generan los animales en pastoreo. Por otro lado, autores como Greenwood et al. (1998), describen que los efectos perjudiciales de la compactación del suelo pueden ser mejorados por la recuperación natural de éste, que incluiría procesos de congelación-deshielo, ciclos de humectación-secado, actividad de la macrofauna (preferentemente por la lombriz de tierra) y la actividad radical.

\section{CONCLUSIONES}

Un incremento en la intensidad de pastoreo durante la temporada primavera - verano no implicó un efecto adverso significativo sobre las propiedades del suelo: densidad aparente, porosidad total, distribución de poros, conductividad hidráulica.

Con respecto a los aspectos funcionales del sistema poroso, la permeabilidad de aire a diferentes tensiones, como también losíndices de continuidad de poros, no presentaron diferencias estadísticas significativas entre tratamientos. 
En todos los casos la mayor capacidad de soporte del suelo, cuando este presentó menor contenido de agua en la temporada primaveraverano, permitió sostener una mayor intensidad de pastoreo sin que la estructura del suelo fuera afectada significativamente.

\section{AGRADECIMIENTOS}

Este estudio fue financiado por la Sociedad Productora de Leche S.A., SOPROLE, en convenio con la Universidad Austral de Chile (160306-01/EN), proyecto "Evaluación de la frecuencia e intensidad de pastoreo sobre atributos productivos y de sustentabilidad en producción de leche". Dorota Dec agradece el financiamiento del proyecto FONDECYT de Postdoctorado 3090038.

\section{BIBLIOGRAFIA}

AHUJA, L.; NANEY, J.; GREEN, R.; NIELSEN, D.; 1984. Macroporosity to characterize spatial variability of hydraulic conductivity and effects of land management. Soil Science Society of America Journal. 48: 699-702.

ALARCON, C. 2008. Efecto de dos intensidades de pastoreo sobre las propiedades físico-hidráulicas de un suelo Trumao durante la temporada primaveraverano. Tesis Licenciado en Agronomía. Valdivia. Universidad Austral de Chile, Facultad de Ciencias Agrarias 68 p.

ALEGRE, J.; PASHANASI, B.; LAVELLE, P. 1996. Dynamics of soil physical properties in Amazonian agroecosystems inoculated with earthworms. Soil Science Society of America Journal 60:1522-1529.

BALL, B.; O‘SULLIVAN, M.; HUNTER, R. 1988. Gas diffusion, fluid flow and derived pore continuity indices in relation to vehicle traffic and tillage. Journal Soil Science 39: 327-339.

BALOCCHI, O.; PULIDO, R.; FERNANDEZ, J. 2002. Comportamiento de vacas lecheras en pastoreo con y sin suplementación con concentrado. Agricultura Técnica 62: 87-98

BAVER, L.; GARDNER, R. 1973. Física de suelos. $1^{\circ}$ Ed. en español, México Ed. UTEHA. 525p.

BEAR, J. 1972. Dynamics of Fluids in Porous Media. Nueva York,Elsevier 764 p.

BLASER, J. 1966. Efecto del animal sobre la pastura. En Turrialba, (ed). Empleo de animales en las investigaciones sobre pasturas. Simposio.
Montevideo Uruguay. 1-24.

CHILE, CENTRO DE INFORMACION DE RECURSOS NATURALES (CIREN) 1999. Descripciones de suelos materiales y símbolos. Estudio agrológico de la provincial de Valdivia, X región, Chile. Santiago. 124 p.

CHILE, CENTRO DE INFORMACIÓN DE RECURSOS NATURALES (CIREN). 2003. Descripciones de suelos materiales y símbolos. Estudio agrológico X Región. Santiago.412 p.

CHAN K.; PRATLEY, J. 1998. Soil structure decline - can the trend be reversed? In 'Agriculture and the environmental imperative'. Pratley J.; Robertson AR. (eds). CSIRO Publishing, Melbourne. pp.129163

DEMANET, R.; CANSECO, C.; REYES, A.; CANTERO, E. 2007.Técnicas de manejo de pastoreo para praderas permanentes. Programa de desarrollo de proveedores. http://lacteos.watts. cl/images/Img_Editor/DocAdjuntos/Boletin\%20 tecnicas\%20de\%20pastoreo.pdf. (Consultado en diciembre, 2009).

DONKOR, N.; GEDIR, J.; HUDSON, R.; BORK, E.;CHANASYK, D.; NAETH,M. 2002. Impacts of grazing systems on soil compaction and pasture production in Alberta. Canadian Journal of Soil Science 82: 1-8.

DÖRNER, J.; HORN, R. 2006. Anisotropy of pore functions in structuraI stagnic luvisols in the weichselian moraine region in Germany. Journal Plant Nutrition Soil Science 169: 212-220.

DÖRNER, J.; DEC, D. 2007. La permeabilidad del aire y conductividad hidráulica saturada como herramienta para la caracterización funcional de los poros del suelo. Revista de la Ciencia del Suelo y Nutrición Vegetal 7: 1-13.

DÖRNER, J.; SCHROEREN, V.; DEC, D.; HORN, R. 2009a. Effect of Land Use on Physical Properties of a Volcanic Soil in South Chile. ISTRO 18th Triennial Conference Proceedings, June 15-19, 2009 Izmir-TURKEY. T4 - 026: 1-6.

DÖRNER, J.; DEC, D.; PENG, X.; HORN, R. 2009b. Efecto del cambio de uso en la estabilidad de la estructura y la función de los poros de un Andisol (Typic Hapludand) del sur de Chile. Journal of Soil Science and Plant Nutrition 9: 190-209.

DREWRY, J.; LITTLE, J.; PATON, R. 2000. A survey of soil physical properties on sheep and dairy farms in southern New Zealand. New Zealand Journal of Agricultural Research. 43:251-258.

EIJKELK AMP AGRISEARCH EQUIPMENT. 2003. Operating instruction. Laboratory permeameters. Geisbeek, Netherlands. 14 p.

ELLIES, A. 1986. Efectos de la consolidación mecánica sobre algunos Distrandeptes y 
Alarcón, C. et al. Intensidad pastoreo, propiedades Andisol...

Palehumultes del sur de Chile. Ciencia del suelo 2:92-97.

ELLIES. A. 1988. Mechanical consolidation in volcanic ash soils. Catena supplement11: 87-92.

ELLIES, A. 2003. Cátedra manejo físico de suelo. Trabajo inédito (no publicado).

ELLIES, A.; GREZ, R.; RAMIREZ, C.1997. La conductividad hidráulica en fase saturada como herramienta para el diagnóstico de la estructura del suelo. Agro Sur 25: 51-56.

ELLIES, A.; HORN, R.; SMITH, R. 2000. Effect of management of a volcanic ash soil on structural properties. Int. Agrophysics 14: 377-384.

FORSYTHE, W. 1974. Manual de laboratorio de física de suelos. Instituto Interamericano de Ciencias Agrícolas de la OEA. Turrialba, Costa Rica. 212 p.

GOIC, L.; MATZNER, M. 1977. Distribución de la Producción de materia seca y características de tres regiones en la zona de las lluvias. Avances en Producción Animal.Universidad de Chile. 2 (1): $23-31$.

GREENWOOD, P.; MCNAMARA, R. 1992: An analysis of the physical condition of two intensively grazed Southland soils. Proceedings of the New Zealand Grassland Association 54: 71-75.

GREENWOOD, K.; MCKENZIE, B. 2001. Grazing effects on soil physical properties and the consequences for pastures: a review. Australian Journal of experimental Agriculture 41: 1231$1250 \mathrm{p}$.

GROENEVELT, P.; KAY, B.; GRANT, C.1984. Physical assessment of a soil with respect to rooting potential. Geoderma 34:101-104.

HARTGE, K. 2000. The effect of soil deformation on physical soil properties-A discourse of the common background, In: Horn, R., van den Akker, J.J.H. Arvidson, J. (eds.). Subsoil compaction. Distribution, processes and consequences. Advances in Geoecology pp 32-43.

HARTGE, K.; HORN, R. 1991. Einführung in die Bodenphysik. Enke. Stuttgart. Germany. 303 p.

HILLEL, D. 1998. Environmental soil physics. San Diego, California , Academic Press 771 p.

JANSSEN, I.; KRUEMMELBEIN, J.; HORN, R.; ELLIES, A. 2004. Physical and hydraulic properties of the Nadi-Soils in south Chile Comparison between untilled and tilled soil. Revista de la Ciencia del Suelo y Nutrición Vegetal 4: $14-28$

MOLDRUP, P.; YOSHIKAWA, S.; OLESEN, T.; KOMATSU, T.; ROLSTON, D. 2001. Air permeability in undisturbed volcanic ash soils predictive model test and soil structure fingerprint. Soil Science Society of America Journal 67: 32 40 .
NISSEN, J.; QUIROZ, C.; SEGUEL, O.; MCDONALD, R.; ELLIES, A. 2006. Flujo hídrico no saturado en Andisoles. Revista de la Ciencia del Suelo y Nutrición Vegetal 6: 9-19.

PARGA, J. 2006. Aspectos claves a considerar en el manejo de pastoreo con vacas lecheras sobre praderas permanentes. http://www.inia. cl/ quilamapu/inproleche/articulosd/manejo\%20 de\%20pastoreo.pdf. (Consultado en diciembre, 2007).

PIZZIO, R.; PALLARES, R. 2000. Manejo del pastoreo y carga animal en pasturas. http:// www.inta.gov.ar/mercedes/info/Pubdiversas/ Jornada\%20pastura\%20pizzio

CargaAnimalMod.pdf. (Consultado en abril, 2008).

RODRIGUEZ, J. 1993. La fertilización de los cultivos: Un método racional. Colección Agricultura, Facultad de Agronomía. Pontificia Universidad Católica de Chile. Santiago. 291 p.

SADZAWKA, A.; CARRASCO, R.; GREZ, R.; MORA, M.; FLORES, H. 2006. Métodos de análisis recomendados para los suelos chilenos. Serie Actas INIA. sp

SOUTHORN, N.; CATTLE, S. 2004.The dynamic of soil quality in livestock grazing systemshttp:// www.regional.org.au/au/asssi/supersoil2004/s14/ oral/1789-southornn.htm. (Consultado en agosto, 2007).

SUAREZ, D. 2003. Uso de la cal en el manejo de suelos ácidos. http://www.tattersall.cl/revista/ rev179/agricola.htm. (Consultado en mayo, 2008).

TABOADA, M. 2007. Efectos del pisoteo y pastoreo animal sobre suelos en siembra directa. Cátedra de Fertilidad y fertilizantes. Trabajo inédito. Facultad de Agronomía. Universidad de Buenos Aires., Argentina. $19 \mathrm{p}$.

TEUBER, N.; PARGA, J.; BALOCCHI, O. 2008. Mejores prácticas de pastoreo. http://www.inia.cl/ medios/biblioteca/ta/NR34744.pdf. (Consultado en diciembre, 2009).

VELASQUEZ, M. 1983. Efecto de la carga animal y contenido de humedad sobre la cohesión y fricción en un suelo Trumao y un suelo Rojo arcilloso de la Décima Región, Chile. Tesis Licenciado en Agronomía. Valdivia. Universidad Austral de Chile, Facultad de Ciencias Agrarias 59 p.

VYHMEISTER, R. 1980. Algunos hídricos aspectos de tres tipos de suelos del sur de Chile. Tesis Licenciado en Agronomía. Valdivia. Universidad Austral de Chile, Facultad de Ciencias Agrarias 59 p.

WILlATT, S.; PULlAR, D.1983. Changes in soil physical properties under grazed pastures. Australian Journal Soil 22: 343-348. 